\title{
Scale and frequency chauvinism in brain dynamics: too much emphasis on gamma band oscillations
}

\author{
Paul L. Nunez $\cdot$ Ramesh Srinivasan
}

Received: 28 July 2010/ Accepted: 7 September 2010/Published online: 2 October 2010

(c) The Author(s) 2010. This article is published with open access at Springerlink.com

In the early 1990s, Wolf Singer advanced a very provocative idea: Different features of an animal's world are represented by distinct cell assemblies that can be bound together by oscillatory synchrony (Singer 1993; Singer and Gray 1995). In particular, simultaneous recordings from animal visual, association, somatosensory, and motor areas reveal that the cortical areas involved in task execution synchronize local field potentials (LFPs) when the animals prepare for the task and focus attention on the relevant stimulus. These findings emphasize LFP oscillations at frequencies in the gamma band (above 20-30 Hz). In perceptual and perceptual-motor tasks, gamma band synchrony is maintained until the task is completed, at which time the coherent patterns collapse and give way to low frequency incoherent oscillatory activity (Singer 2001). This "binding by synchrony" idea may well qualify as "a paradigm shift" in neuroscience by suggesting that coherent oscillatory activity across different cell assemblies is a critical feature underlying cognition rather than just fixed anatomical connections (Buzsaki 2006). This letter does not challenge the importance of gamma oscillations, nor do we deny the compelling notion of binding by synchrony. Rather, we question the (perhaps implicit) assumption by many neuroscientists that the most important aspects of human cognition are based mainly or even exclusively on the catch-all category labeled "gamma oscillations." For one thing, the theoretical idea proposed by Singer that synchronization facilitates binding does not

P. L. Nunez $(\bowtie)$

Cognitive Dissonance, LLC, Covington, LA, USA

e-mail:pnunez@tulane.edu

R. Srinivasan

Department of Cognitive Science,

University of California at Irvine, Irvine, CA, USA depend on or specify any particular frequency band. Our objections to the extreme gamma focus are based on several interrelated arguments as follows.

Our first argument comes from LFP data collected in monkeys and cats that reveal complex dynamics in multiple frequency bands and their relationship to cognitive processing. Oscillations at lower frequencies have been observed in LFPs in animal models in response to sensory stimulation, especially at alpha band $(8-13 \mathrm{~Hz})$ frequencies (Dinse et al. 1997; Steriade et al. 1990; Lopes da Silva 1991; Rougeul-Buser and Buser 1997). Discounting the notion that low frequency rhythms reflect idling of cortical neurons, studies in cats and monkeys have demonstrated low frequency oscillatory synchronization across cortical areas during perceptual and motor tasks (von Stein et al. 2000; Roelfsema et al. 1997). These oscillations are potentially related to top-down signaling reflecting expectancy and attentional focus (Riehle et al. 1997; Engel et al. 2001). Thus, we may anticipate substantial potential information at lower LFP frequencies in animal models that has yet to be thoroughly investigated in relation to cognitive processing. While gamma band oscillations no doubt play a significant role in animal brains, oscillatory synchronization in other frequency bands is easily observed and could play a critical role in "binding by synchrony" or its generalization, "binding by resonance," discussed at the end of this letter. We are unaware of anything preventing full characterization of LFP spectra in animal models rather than emphasizing only gamma band oscillations. One caveat, however, is that the alpha band and slower oscillations that dominate human scalp potentials may be substantially facilitated by long ( $\sim 30 \mathrm{~ms}$ ) axon delays (Nunez 1995, 2010a, b; Nunez and Srinivasan 2006a, b). Axon delays in small mammals are apparently much shorter, potentially limiting the importance of certain slower 
frequencies (e.g., global alpha) in animal studies, but such limitation should not apply to all slow frequencies (e.g., local theta and local alpha).

Our second and perhaps most convincing argument concerns the extensive studies of human scalp potentials (EEG) obtained since the first recordings in the 1920s. These have revealed numerous robust correlations between multiple brain states or cognitive tasks with EEG dynamic behavior, and nearly all recorded power in scalp potentials is lower than $15-20 \mathrm{~Hz}$. The sheer volume of this work revealing numerous confirmed conscious correlates completely dwarfs all existing gamma studies and provides the basis for nearly all clinical EEG (Niedermeyer and Lopes da Silva 1995). Included in the voluminous classical EEG work are numerous studies showing selective cognitive correlates of distinct sleep stages, anesthesia depth, severity of coma, and many task-related correlations to delta, theta, and alpha band oscillations. Here, we mention only a few relevant studies. Theta, lower alpha, and upper alpha band long range $(10-25 \mathrm{~cm})$ scalp coherence patterns are selectively correlated with the performance of mental tasks (Nunez 1995; Nunez et al. 1997, 1999, 2001; Sauseng et al. 2005). In some studies of steady-state visually evoked potentials (SSVEPs), subjects are presented with problems superimposed on a $13 \mathrm{~Hz}$ light flicker (Silberstein et al. 2001, 2003, 2004; Silberstein 1995). Large inter-site coherences peak about $2 \mathrm{~s}$ after problem presentation. These findings again suggest formation of large-scale $(5-25 \mathrm{~cm})$, alpha band cortical networks associated with solving several different kinds of problems. Additional robust cognitive correlates are provided by event-related potentials (ERPs), which include the widely studied P300; these waveforms have dominant power in the theta and alpha bands (Klimesch et al. 2007). Alan Gevins and colleagues have recorded evoked potential covariance patterns (correlations between scalp evoked potential waveforms as a function of time lag, typically $30-80 \mathrm{~ms}$ ) over more than 30 years (Gevins et al. 1983, 1987, 1997), suggesting the formation of very large-scale $(5-25 \mathrm{~cm})$ preparatory networks that predict performance accuracy just before the task is carried out.

Perhaps, most closely related to Singer's original work on perceptual binding are studies of conscious perception during binocular rivalry using SSVEPs (Srinivasan et al. 1999; Srinivasan and Petrovic 2006). In these studies, incongruent (theta or alpha sub-band) flickering stimuli are presented one to each eye while conscious perception alternates between the images presented to each eye. A more integrated scalp coherence pattern at each frequency occurs with conscious perception of the stimulus flickering at the matching frequency. Conscious perception, in these experiments, occurs with enhanced dynamic "binding" of the brain hemispheres within the theta or alpha frequency band. In addition, it should be noted that dynamic processes occurring in other frequency bands can remain "unbound," allowing other brain networks to engage in independent actions. In a subsequent experiment (Sutoyo and Srinivasan 2009), four flickering stimuli (in the lower and upper alpha band) were presented two to each eye; within each eye the two flickers were presented in different hemifields. At any time, the subject reported awareness of two flickers, one in each hemifield. In addition to SSVEP responses at the flicker frequencies, nonlinear responses at the sum of the two flicker frequencies (in the $18-22 \mathrm{~Hz}$ range) were observed. These nonlinear responses of neural populations receiving input from both hemifields may reflect binding of the visual hemifields into a coherent percept. These nonlinear beta band responses were shown to be independent of the subject's conscious perception, while responses to the two flickers in both lower and upper alpha band showed increased synchronization with conscious perception.

Our third point concerns an unjustified tendency to downplay large-scale scalp data in favor of intracranial data, in other words, "scale-chauvinism." If, as we contend here, brains are genuine complex systems, the common view that sparsely recorded intracranial data somehow provide a gold standard for scalp recordings appears quite misguided. Consider the following thought experiment. Cover a $20 \times 20 \mathrm{~cm}$ dura surface with 10,000 1-mm diameter electrodes with $1-\mathrm{mm}$ interelectrode spacings. The signals obtained from (appropriately) weighted averages over $6 \times 6 \mathrm{~cm}$ and $2 \times 2 \mathrm{~cm}$ sub-regions are expected to closely match unprocessed scalp potentials and scalp Laplacians, respectively (Nunez et al. 2001; Nunez and Srinivasan 2006a). Because cortical source behavior is different in different temporal frequency bands, we generally expect EEG and ECoG temporal spectra to differ across these three observational scales. In other words, selective temporal filtering is expected to occur as a byproduct of the spatial filtering caused by either averaging over dura signals (in our thought experiment) or volume conduction (in scalp recordings). This prediction is well supported by experiments; temporal spectra are indeed scale-dependent. For example, beta and gamma frequencies are largely absent at the scalp, and the widely synchronous part of the alpha band is expected to be much weaker in dura recordings (because the influence of $\mathrm{cm}$ scale source synchrony on amplitude is expected to be much lower in dura recordings). Furthermore, the largescale alpha coherence patterns closely related to mental tasks cannot be observed with strictly local dura recordings. Consider similar arguments applied to even smaller scales. Each signal from the meso (intermediate)-scale dura electrodes represents a space average over LFPs in the cortical depths caused by both the physical separation from 
cortical sources and non-zero electrode size. Such LFPs could provide a gold standard for observed dura potentials only if millions of micro-scale electrodes were employed in the cortical depths. Similarly, dura potentials could provide an approximate gold standard for scalp potentials only if thousands of $\mathrm{mm}$ scale electrodes spread over hundreds of $\mathrm{cm}^{2}$ were employed. Only then could scalp potential dynamics (frequency spectra, amplitude maps, coherence patterns, etc.) be accurately predicted from ECoG recordings.

Synchronization and "binding" were known to human EEG scientists long before the discovery of gamma oscillations in animal models. Why did synchrony and oscillations only become important when they were reported in the gamma band in animal models? As a partial answer, we suggest a common error in thinking about brains-the failure to account for relations between spatial and temporal scales that might be generally expected in complex systems. Thus, as outlined in the previous paragraph, the very large-scale EEG shows a different spectral signature than the (intermediate scale) high-resolution EEG (Laplacian or dura imaging) (Nunez et al. 2001; Nunez and Srinivasan 2006a); the temporal dynamics of ECoG (cortex) differs from both the first two measures (Pfurtscheller and Cooper 1975). Furthermore, we expect intracranial data to be fractal-like with observed dynamics dependent on electrode size (reviewed in Nunez 1995). The transition between intracranial animal work and human (scalp) EEG studies involves two critical experimental changes, animal species and spatial measurement scale; the distinct spectral changes due to these two differences may be difficult to distinguish. We note that the large-scale $(5-25 \mathrm{~cm})$ theta and alpha coherence patterns observed in humans cannot occur in small mammals, suggesting important species differences in the dynamics of cognition. In particular, the dominance of large-scale, low frequency oscillations in humans is apparently at least partly due to long axonal delays absent in small mammals. That is, the human alpha band consists of multiple phenomena, including globally coherent rhythms (perhaps standing and traveling waves) and more localized activity (perhaps thalamocortical networks). These distinct alpha rhythms, which may or may not have overlapping frequencies, have been distinguished by reactivity, coherence patterns over the scalp, and spatial filtering by the Laplacian (Andrew and Pfurtscheller 1996, 1997; Pfurtscheller et al. 1996; Nunez and Srinivasan 2006a, b, 2007; Nunez 2010a, b). The implication is that human scalp EEG is not merely a poor man's version of intracranial data, but instead contains different kinds of information about neocortical dynamics at very larger scales that would be difficult or impossible to observe in intracranial ECoG recordings or animal models. Thus, we suggest that an experimental design aimed to investigate gamma band oscillations exclusively implies a priori mischaracterization of the overall brain dynamics, which can look quite different when observed at different spatial scales.

Our fourth objection concerns gamma oscillations recorded in human scalp EEG. Substantial interpretation problems occur for scalp-recorded frequencies higher than about 15 or $20 \mathrm{~Hz}$, which are difficult if not impossible to distinguish from muscle and other artifact. When one actually views a genuine EEG recording, it quickly becomes obvious that even "small" purposely generated muscle-related beta and gamma oscillations have much larger amplitudes than whatever high frequency brain signals might actually reach the scalp. This limitation has been anticipated by an observed absence of global gamma synchrony in human ECoG recordings (Menon et al. 1996), where the cortical domains of spatially correlated activity underlying perceptual categorization in human gamma band ECoG $(20-50 \mathrm{~Hz})$ were limited to less than 1 or $2 \mathrm{~cm}$ diameters, and only intermittent synchronization was observed. In addition, we note recent studies that have questioned the cortical origins of gamma rhythms recorded on the scalp. In one study, drug-induced muscle paralysis (excepting the dominant arm) was used to compare preand post-paralysis EEG and evoked potentials with subjects placed in varied conditions (Whitham et al. 2007). EEG power in bands above $20 \mathrm{~Hz}$ was reduced by factors of between 10 and 200 between pre- and post-paralysis. These enormous power reductions were widespread, not limited to electrode sites close to scalp muscles. In another study, gamma rhythms recorded on the scalp were reported to be closely related to microsaccades rather than cortical activity (Yuval-Greenberg et al. 2008). Moreover, this type of artifact can also be observed in intracranial recordings with implanted electrodes in human subjects (Jerbi et al. 2009). We acknowledge that brain sources may contribute small beta and gamma power to scalp EEG as indicated in a combined EEG/ECoG study (Ball et al. 2008). Perhaps, MEG or improved technical methods in EEG can avoid some of the artifact contamination; nevertheless, extracranial studies of gamma oscillations are severely limited by technical barriers. In the absence of strong independent contrary evidence, we suggest that gamma band potentials recorded on the scalp are severely contaminated with artifact. While this limitation does not apply directly to intracranial studies, it does suggest that some EEG scientists may have been "seduced" by intracranial studies to emphasize gamma in scalp recordings, rather than the much more robust and reliable lower frequency bands.

We argue that generally in complex adaptive systems, for which brains provide the pre-eminent examples, interesting dynamics is to be expected at multiple observational scales, implying that important conscious correlates are 
also to be expected at multiple scales. This prediction is well supported by the (large scale) human scalp data cited above showing the importance of low frequencies to cognition together with the (smaller scale) human gamma band ECoG data also related to cognition (Menon et al. 1996; Crone et al. 2001; Meador et al. 2002; Supratim et al. 2008). In particular, Canolty et al. (2006) observed robust coupling between the high and low frequency bands of human ECoG. The low-frequency theta $(4-8 \mathrm{~Hz})$ phase was observed to modulate power in the high gamma $(80-150 \mathrm{~Hz})$ band, with stronger modulation occurring at higher theta amplitudes. Different behavioral tasks evoked distinct patterns of theta/high gamma coupling across the cortex. The results imply that transient coupling between low and high frequency brain rhythms may coordinate activity in distributed cortical areas, suggesting a mechanism for effective inter-network communication during cognitive processing in humans.

Our final issue concerns the original binding due to synchronization hypothesis advanced by Singer; our related theoretical suggestion follows partly from taking the idea of complex brains quite seriously. Mathematical studies of (very broadly defined) "coupled oscillators" suggest that the now familiar "binding by synchrony" might be viewed as a sub-category of a much broader phenomenon, "binding by resonance," in which cell assemblies selectively interact in multiple "matching" frequency ranges that need not be equal. Cognitive processes and even consciousness itself appear to be correlated with EEG oscillations in multiple, but selective frequency bands. With this in mind, mathematicians engaged in neuroscience research (Hoppensteadt and Izhikevich 1998; Izhikevich 1999) showed that weakly connected (non-chaotic) oscillators are expected to interact strongly only when their characteristic (resonant) frequencies obey certain resonant relations. The most familiar example occurs when two oscillators have identical resonant frequencies, but selective interaction also occurs for other frequency combinations. These "oscillators" are sufficiently general to be associated with either networks or global synaptic fields (Nunez and Srinivasan 2006a, b, 2007; Nunez 2010a, b). A simple example is the case in which global synaptic fields oscillating at frequency $f_{0}$ (perhaps theta or alpha) can cause strong coupling between two isolated (perhaps gamma) networks with characteristic frequencies $f_{1}$ and $f_{2}$, e.g., strong interaction is predicted when $f_{0}=f_{1} \pm f_{2}$. This theoretical result and the EEG, ECoG, and LFP data support a conceptual framework where cognitive processing depends critically on resonance phenomena in multiple select frequency bands. In humans, at least, such select frequencies can apparently cover a very broad range (maybe something like $1-150 \mathrm{~Hz}$ ), although specific experimental designs, especially spatial measurement scale, are likely to reveal only (perhaps small) parts of this spectral content. Perhaps, properly tuned brains orchestrate the beautiful music of sentience, but brain music is certainly not limited to the currently popular gamma "tunes." As noted quite succinctly in a recent editorial on measuring cortical activity, maybe one should expect to find only what one is looking for (Jacobs 2010).

Open Access This article is distributed under the terms of the Creative Commons Attribution Noncommercial License which permits any noncommercial use, distribution, and reproduction in any medium, provided the original author(s) and source are credited.

\section{References}

Andrew C, Pfurtscheller G (1996) Event-related coherence as a tool for studying dynamic interaction of brain regions. Electroencephalogr Clin Neurophysiol 98:144-148

Andrew C, Pfurtscheller G (1997) On the existence of different alpha band rhythms in the hand area of man. Neurosci Lett 222:103-106

Ball T, Demandt E, Mutschler I, Neitzel E, Mehring C, Vogt K, Aertsen A, Schulze-Bonhage A (2008) NeuroImage 41:302-310

Buzsaki G (2006) Rhythms of the brain. Oxford University Press, New York

Canolty RT, Edwards E, Dalal SS, Soltani M, Nagarajan SS, Kirsch HE, Berger MS, Barbaro NM, Knight RT (2006) High gamma power is phase-locked to theta oscillations in human neocortex. Science 313:1626-1628

Crone NE, Boatman D, Gordon B, Hao L (2001) Induced electrocorticographic gamma activity during auditory perception. Clin Neurophysiol 112:565-582

Dinse HR, Kruger K, Akhavan AC, Spengler F, Schoner G, Schreiner CE (1997) Low-frequency oscillations of visual auditory and somatosensory cortical neurons evoked by sensory stimulation. Int J Psychophysiol 26:205-227

Engel AK, Fries P, Singer W (2001) Dynamic predictions: oscillations and synchrony in top-down processing. Nat Rev Neurosci 2:704-716

Gevins AS, Schaffer RE, Doyle JC, Cutillo BA, Tannehill RL, Bressler SL (1983) Shadows of thought: shifting lateralization of human brain electrical patterns during brief visuomotor task. Science 220:97-99

Gevins AS, Morgan NH, Bressler SL, Cutillo BA, White RM, Illes J, Greer DS, Doyle JC, Zeitlin GM (1987) Human neuroelectric patterns predict performance accuracy. Science 235:580-585

Gevins AS, Smith ME, McEvoy L, Yu D (1997) High resolution EEG mapping of cortical activation related to working memory: effects of task difficulty, type of processing, and practice. Cereb Cortex 7:374-385

Hoppensteadt FC, Izhikevich EM (1998) Thalamo-cortical interactions modeled by weakly connected oscillators: could brain use FM radio principles? Biosystems 48:85-92

Izhikevich EM (1999) Weakly connected quasi-periodic oscillators, FM interactions, and multiplexing in the brain. SIAM J Appl Math 59:2193-2223

Jacobs J (2010) Measuring cortical activity-we will only detect what we are looking for. Clin Neurophysiol 121:268-269

Jerbi K, Freyermuth S, Dalal S, Kahane P, Bertrand O, Berthoz A, Lachaux JP (2009) Saccade related gamma-band activity in intracerebral EEG: dissociating neural from ocular muscle activity. Brain Topogr 22:18-23 
Klimesch W, Hanslmayr S, Sauseng P, Gruber W, Doppelmayr M (2007) The P1 and travelling alpha waves: evidence for evoked oscillations. J Neurophysiol 97:1311-1318

Lopes da Silva FH (1991) Neuronal mechanisms underlying brain waves. Electroencephalogr Clin Neurophysiol 79:81-93

Meador KJ, Ray PG, Echauz JR, Loring DW, Vachtsevanos GJ (2002) Gamma coherence and conscious perception. Neurology $59: 847-854$

Menon V, Freeman WJ, Cutillo BA, Desmond JE, Ward MF, Bressler SL, Laxer KD, Barbaro N, Gevins AS (1996) Spatio-temporal correlations in human gamma band electrocorticograms. Electroencephalogr Clin Neurophysiol 98:89-102

Niedermeyer E, Lopes da Silva FH (eds) (1995) Electroencephalography. Basic principals, clinical applications, and related fields, 5th edn. Williams and Wilkins, London

Nunez PL (1995) Neocortical dynamics and human EEG rhythms. Oxford University Press, New York

Nunez PL (2010a) Brain, mind, and the structure of reality. Oxford University Press, New York

Nunez PL (2010b) Implications of white matter correlates of EEG standing and traveling waves. NeuroImage (in press)

Nunez PL, Srinivasan R (2006a) Electric fields of the brain: the neurophysics of EEG. Oxford University Press, New York

Nunez PL, Srinivasan R (2006b) A theoretical basis for standing and traveling brain waves measured with human EEG with implications for an integrated consciousness. Clin Neurophysiol 117:2424-2435

Nunez PL, Srinivasan R (2007) Hearts don't love and brains don't pump: neural correlates of conscious experience. J Conscious Stud 14:20-34

Nunez PL, Srinivasan R, Westdorp AF, Wijesinghe RS, Tucker DM, Silberstein RB, Cadusch PJ (1997) EEG coherency. I. Statistics, reference electrode, volume conduction, Laplacians, cortical imaging, and interpretation at multiple scales. Electroencephalogr Clin Neurophysiol 103:499-515

Nunez PL, Silberstein RB, Shi Z, Carpenter MR, Srinivasan R, Tucker DM, Doran SM, Cadusch PJ, Wijesinghe RS (1999) EEG coherency II: experimental comparisons of multiple measures. Electroencephalogr Clin Neurophysiol 110:469-486

Nunez PL, Wingeier BM, Silberstein RB (2001) Spatial-temporal structure of human alpha rhythms: theory, microcurrent sources, multiscale measurements, and global binding of local networks. Hum Brain Mapp 13:125-164

Pfurtscheller G, Cooper R (1975) Frequency dependence of the transmission of the EEG from cortex to scalp. Electroencephalogr Clin Neurophysiol 38:93-96

Pfurtscheller G, Stancak A Jr, Neuper C (1996) Event-related synchronization (ERS) in the alpha band-an electrophysiological correlate of cortical idling: a review. Int J Psychophysiol 24:39-46

Riehle A, Gruen S, Diesmann M, Aertsen A (1997) Spike synchronization and rate modulation differentially involved in motor cortical function. Science 278:1950-1953

Roelfsema PR, Engel AK, Konig P, Singer W (1997) Visuomotor integration is associated with zero time-lag synchronization among cortical areas. Nature 385:157-161
Rougeul-Buser A, Buser P (1997) Rhythms in the alpha band in cats and their behavioral correlates. Int J Psychophysiol 26:191-293

Sauseng P, Klimesch W, Schabus M, Doppelmayr M (2005) Frontoparietal EEG coherence in theta and upper alpha reflect central executive functions of working memory. Int J Psychophysiol 57:97-103

Silberstein RB (1995) Steady-state visually evoked potentials, brain resonances, and cognitive processes. In: Nunez PL (au) Neocortical dynamics and human EEG rhythms. Oxford University Press, New York, pp 272-303

Silberstein RB, Nunez PL, Pipingas A, Harris P, Danieli F (2001) Steady state visually evoked potential (SSVEP) topography in a graded working memory task. Int J Psychophysiol 42:219-232

Silberstein RB, Danieli F, Nunez PL (2003) Fronto-parietal evoked potential synchronization is increased during mental rotation. NeuroReport 14:67-71

Silberstein RB, Song J, Nunez PL, Park W (2004) Dynamic sculpting of brain functional connectivity is correlated with performance. Brain Topogr 16:240-254

Singer W (1993) Synchronization of cortical activity and its putative role in information processing and learning. Annu Rev Physiol 55:349-374

Singer W (2001) Consciousness and the binding problem. Ann N Y Acad Sci 929:123-146

Singer W, Gray (1995) Visual feature integration and temporal correlation hypotheses. Annu Rev Neurosci 8:555-586

Srinivasan R, Petrovic S (2006) MEG phase follows conscious perception during binocular rivalry induced by visual stream segregation. Cereb Cortex 16:597-608

Srinivasan R, Russell DP, Edelman GM, Tononi G (1999) Frequency tagging competing stimuli in binocular rivalry reveals increased synchronization of neuromagnetic responses during conscious perception. J Neurosci 19:5435-5448

Steriade M, Gloor P, Llinas RR, Lopes da Silva FH, Mesulam MM (1990) Basic mechanisms of cerebral rhythmic activities. Electroencephalogr Clin Neurophysiol 76:481-508

Supratim R, Niebur E, Hsiao SS, Sinai A, Crone NE (2008) Highfrequency gamma activity $(80-150 \mathrm{~Hz})$ is increased in human cortex during selective attention. Clin Neurophysiol 119:116-133

Sutoyo D, Srinivasan R (2009) Nonlinear SSVEP responses are sensitive to the perceptual binding of the visual hemifields during conventional "eye" rivalry and interocular "percept" rivalry. Brain Res 1251:245-255

von Stein A, Chiang C, Konig P (2000) Top-down processing mediated by intra-areal synchronization. Proc Natl Acad Sci USA 97:14748-14753

Whitham EM, Pope KJ, Fitzgibbon SP, Lewis T, Clark CR, Loveless S, Broberg M, Wallace A, DeLosAngeles D, Lillie P, Hardy A, Fronskof R, Pulbrook A, Willoughby JO (2007) Scalp electrical recording during paralysis: quantitative evidence that EEG frequencies above $20 \mathrm{~Hz}$ are contaminated by EMG. Clin Neurophysiol 118:1877-1888

Yuval-Greenberg S, Tomer O, Keren AS, Nelken I, Deouell LY (2008) Transient induced gamma-band response in EEG as a manifestation of miniature saccades. Neuron 58:429-441 